\title{
Repensar a Ciatalgia Perante Variação Anatómica Bilateral do Nervo Isquiático, com Origem Baixa e Divisão Alta: Importância Histórica, Anatómica e Clínica
}

\author{
Rethinking Sciatica In View of a Bilateral Anatomical \\ Variation of the Sciatic Nerve, with Low Origin and High \\ Division: Historical, Anatomical and Clinical Approach
}

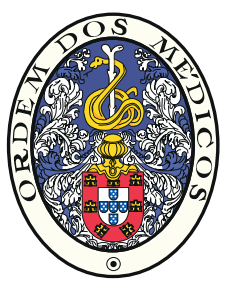

Fernando Silva RIBEIRO ${ }^{1}$, Maria Alexandre BETTENCOURT PIRES $\square^{2,3}$, Edivaldo Xavier da SILVA JÚNIOR ${ }^{1}$, Diogo CASAL 2,3, Daniel CASANOVA-MARTINEZ ${ }^{4}$, Diogo PAIS ${ }^{2,3}$, João Erse GOYRI-O'NEILL ${ }^{3}$

Acta Med Port 2018 Oct;31(10):568-575 - https://doi.org/10.20344/amp.10567

\section{RESUMO}

Introdução: O objectivo do presente estudo é o relato de um caso de variação anatómica do nervo isquiático, por origem baixa e divisão alta bilateral.

Material e Métodos: Foi dissecado um cadáver feminino de 66 anos, conservado pelas técnicas originais desenvolvidas no nosso departamento.

Resultados: No trabalho regular de dissecção cadavérica da disciplina de Anatomia Regional II detectou-se nervo isquiático, em ambos os membros, com pequeno trajecto, de aproximadamente, $8-10 \mathrm{~cm}$, dividido em nervos fibular comum e tibial, ao nível da margem inferior do músculo glúteo máximo.

Discussão: Casos semelhantes aos do presente estudo, foram descritos por alguns autores desde Leonardo da Vinci, Da Cortona, ou Eustachius, demonstrando a importância desse conhecimento, tanto em termos clínicos, como cirúrgicos ou anestesiológicos. Diversas ilações são possíveis, por revisão do presente caso, infrequente.

Conclusão: Para além de proporcionar conhecimento prévio da anatomia loco-regional, o estudo das variações do nervo isquiático orienta o melhor seguimento de patologias, bem como acessos cirúrgicos e/ou anestésicos. Por mais meticulosos que sejam os nossos estudos anatómicos, jamais poderemos considerar terminada ou definitiva a investigação em anatomia humana.

Palavras-chave: Cadáver; Nervo Isquiático/anatomia

\section{ABSTRACT}

Introduction: The aim of the present study is to report an original, unusual, case of bilateral anatomical variation of the sciatic nerve, with low origin and high division.

Material and Methods: Anatomical dissection was performed on a 66 year-old female cadaver. The corpse was embalmed and conserved through our original embalming techniques.

Results: The particular anatomical variation was first detected during routine dissection classes for undergraduate students. The study was completed with contralateral dissection to unveil bilateral variation. In both hind limbs, the sciatic nerve had a low origin, deep below the mid-gluteal region, and suffered high division, near the lower margin of the gluteal region, after a short length of circa $8 \mathrm{~cm}$, to divide into the common fibular and tibial nerves.

Discussion: We detect several cases of sciatic nerve high division, in the reports of the earliest anatomists, such as Leonardo da Vinci, Vesalius, Da Cortona, or Eustachius. Such ancestral interest for these anatomical variations demonstrates the importance of their knowledge for health professionals of different areas.

Conclusion: The accurate study of sciatic nerve anatomical variations bears evident surgical, anaesthesiology and clinical applications. As more meticulous as our anatomical studies may get, one will never reach the state of perfection to consider such studies as definitive.

Keywords: Cadaver; Sciatic Nerve/anatomy

\section{INTRODUÇÃO}

$\mathrm{Na}$ sequência do artigo de revisão anteriormente publicado na Acta Médica Portuguesa, ${ }^{1}$ os autores apresentam a adição de um novo caso original de origem baixa e divisão alta bilateral do nervo isquiático não antes classificado. O mais antigo caso publicado, de divisão alta do nervo isquiático, data de 1618.

As épocas em que mais se realizou dissecção humana, coincidem num breve prazo com épocas áureas de desenvolvimento da cultura em geral e de florescimento de grandiosos empreendimentos e descobertas científicas (pensamos em particular na era dos Egípcios, na civilização grega ou mais recentemente, no Renascimento). O conhecimento aprofundado do corpo humano serve como impulsionador do desenvolvimento de curiosidade científica e do melhor raciocínio científico (sendo reciprocamente verdadeiro, o inverso....). ${ }^{2}$ No polo oposto, aquelas épocas em que a dissecção humana foi banida ou negligenciada pelas mais variadas razões, religiosas ou culturais, correspondem a períodos em que os conhecimentos e a cultura humana sofreram atraso do desenvolvimento ou

1. Departamento de Fisioterapia. LABEPAH - Laboratório de Estudo e Pesquisa em Anatomia Humana. Universidade de Pernambuco. Pernambuco. Brasil.

2. Departamento de Anatomia. Nova Medical School. Universidade Nova de Lisboa. Lisboa. Portugal.

3. Associação Anatómica Portuguesa. Lisboa. Portugal.

4. Escuela de Medicina. Universidad de Valparaiso. San Felipe. Chile.

$\triangle$ Autor correspondente: Maria Alexandre Bettencourt Pires. alexandra.pires@nms.unl.pt

Recebido: 25 de março de 2018 - Aceite: 11 de julho de 2018 | Copyright @ Ordem dos Médicos 2018 
fraca visibilidade científica, como durante a época medieval. ${ }^{3}$ Modernamente, após um estranho retrocesso no final do séc. XX, começamos agora a verificar recrudescimento da noção da factual importância da Anatomia e da dissecção, como base fundamental dos estudos médicos. ${ }^{4-11}$

$\mathrm{Na}$ NOVA Medical School de Lisboa, temos mantido desde a primeira hora, o hábito regular de dissecção humana, tanto para estudos pré-graduados em Medicina, como para pós-graduação e cursos de especialização e/ ou treino cirúrgico (cursos hands-on). ${ }^{12,13}$ Para esse efeito, temos vindo a promover e manter uma listagem legalizada de doações cadavéricas humanas, bem como esforços de inovadora modernização das técnicas de embalsamento, pela introdução de um sistema de perfusão por propulsão intermitente que permite melhor impregnação dos capilares sanguíneos, e consequente melhor fixação e conservação cadavérica. ${ }^{14} \mathrm{Em}$ consequência dos bons resultados obtidos com as estas inovações, temos vindo a receber pedidos por parte de anatomistas internacionais que desejam aprender essas técnicas de conservação e também juntar-

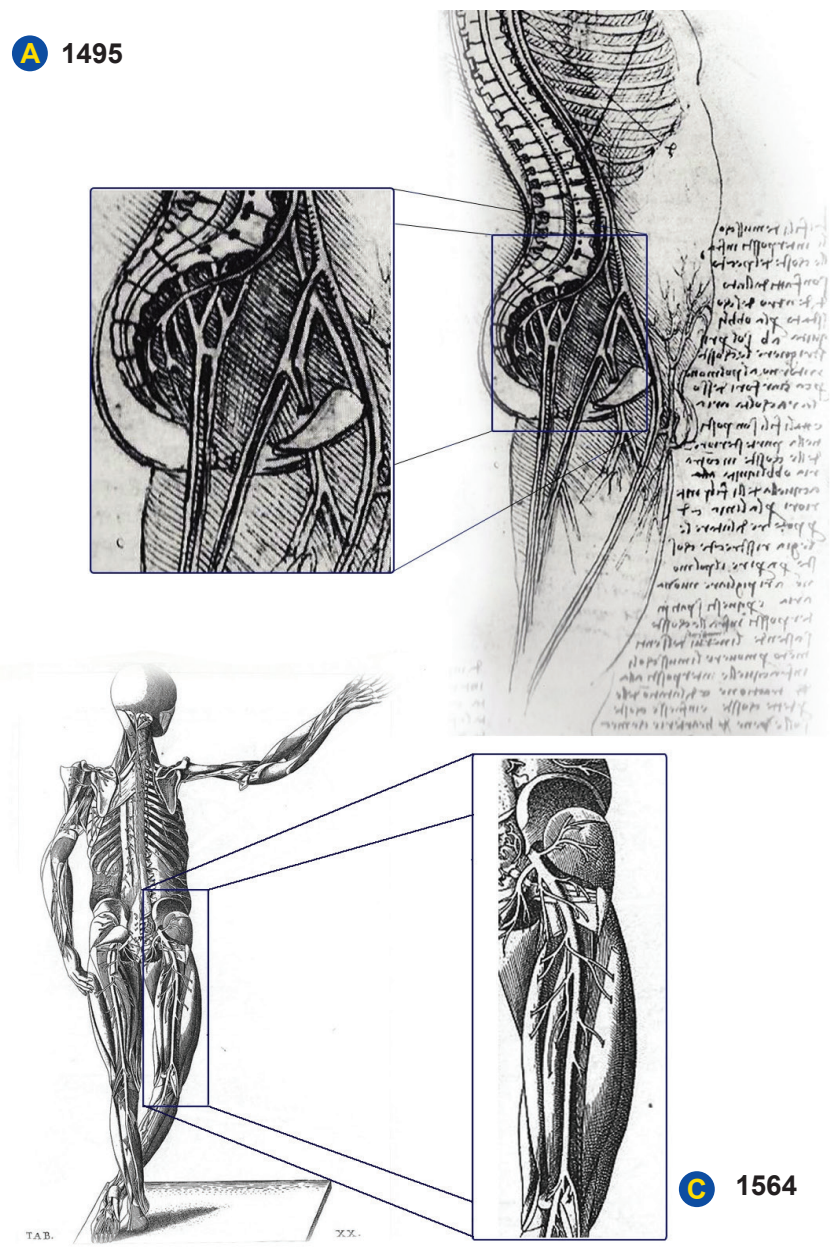

se às nossas equipas de trabalho para aprimorar o treino pessoal de dissecção. É esse o caso dos dois investigadores e co-autores, da Universidade de Pernambuco, Brasil, e da Universidade de Valparaiso, Chile, que visitaram o departamento com bom usufruto de toda a qualidade do material cadavérico português, culminando esse trabalho na investigação utilizada para os presentes trabalhos.

\section{Contexto histórico}

Desde 1490, com Leonardo Da Vinci (Fig. 1A), ${ }^{15}$ ficou bem estabelecida a origem do nervo isquiático, de ramos do plexo sagrado e IV ramo lombar.

Em 1581, Ambroise Paré ${ }^{16}$ descreve com impressionante detalhe, o trajeto do nervo isquiático, em relação com a importância do quadro clínico da ciatalgia. Na pg. XCIIII (94) do seu III Livro, Paré aponta, numa imagem da região glútea, "o ponto da articulação da anca onde se devem aplicar remédios próprios à cura da 'gota isquiática". ${ }^{16}$

Pietro Da Cortona ${ }^{17}$ (Fig. 1B) representa imagem anatómica de um caso de nervo isquiático muito semelhante
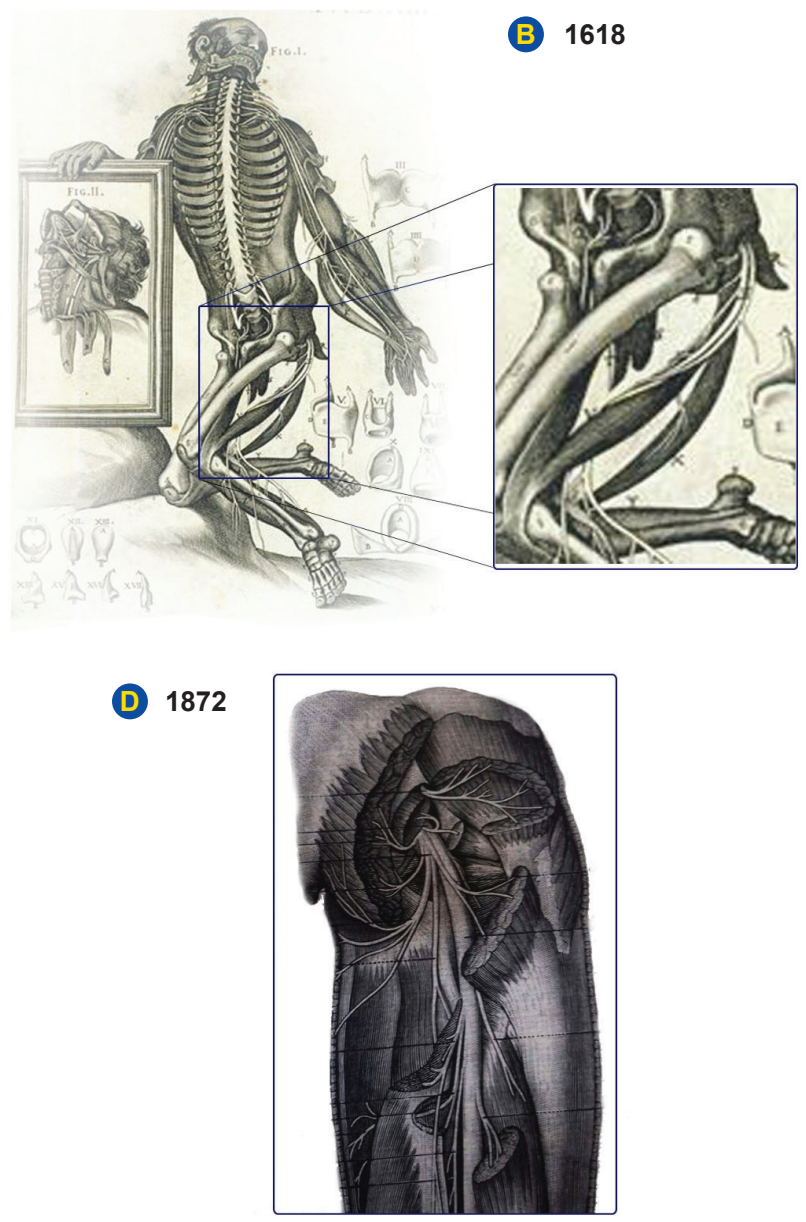

Figura 1 - Esboço Histórico da Anatomia do nervo Isquiático. A: 1495 - Leonardo da Vinci [Windsor Collection - Q IV f. 9 r/Clark 19114 r/c.1495 - 1499]: Representou os quatro ramos de origem do nervo, a par da ramificação terminal da aorta abdominal. A imagem apresenta duplicação do nervo isquiático em todo o seu trajecto; B: 1618 - Pietro Da Cortona oferece-nos imagem de dissecção de todo o trajecto do nervo isquiático, dividido em dois ramos, desde a origem, até ao ápice da região poplítea, onde se afastam, por separação, os dois nervos tibial e fibular comum; C: 1564 - Bartolommeo Eustachius: Representou a origem e trajecto do nervo, de acordo com o tipo classificativo mais frequente; D: 1872 - Ph. Sappey apresenta imagem de dissecção glútea do nervo isquiático e seus ramos, de acordo com o tipo classificativo mais frequente $(85 \%)$. 
àquele que aqui apresentamos, 400 anos depois. No entanto, nas Tabulae XVII e XIX, dedicadas aos nervos espinhais posteriores, Da Cortona representa um nervo isquiático indiviso até ao vértice da região poplítea, e com origem profundamente ao musculo piriforme, correspondendo plenamente ao tipo classificativo mais corrente (85\%). Estes pioneiros anatomistas representaram casos de duplicação do tronco do nervo isquiático, em figuração do processo de separação dos elementos primitivos do nervo. Por sua vez, em 1564, Bartolommeo Eustachius ${ }^{18}$ (Fig. 1C), assim como mais tarde Ph. Sappey ${ }^{19}$ (Fig. 1D), ou J. Cruveilhier, ${ }^{20}$ representam a origem, trajecto e divisão do nervo, de acordo com o tipo classificativo mais frequente na literatura médica mundial. Ambos apontam o facto de o nervo isquiático se poder dividir a qualquer nível, desde o plexo sagrado até à fossa poplítea. A propósito da hipótese de divisão alta do nervo Isquiático, Sappey (1867-72) refere, acerca dos ramos de divisão: "Estes dois nervos, simplesmente acolados/unidos um ao outro, podem com frequência separar-se em pontos mais elevados, e por vezes mesmo, perto da origem do nervo isquiático". 19

Beaton e Anson ${ }^{21}$ propuseram em 1937 a classificação em seis grupos, das principais variações anatómicas do nervo isquiático. No tipo classificativo mais frequente, observaram em $85 \%$ dos casos, o nervo isquiático indiviso, nascendo abaixo do músculo piriforme. E em $3 \%$ dos casos, observaram origem e divisão abaixo do músculo piriforme, como no presente caso em análise.

\section{Aplicação clínica}

O conhecimento da origem, trajecto e variações morfológicas do nervo isquiático e da sua relação com as estruturas anatómicas adjacentes são essenciais para fundamentar a prática clínica, baseada em evidências. Nesse sentido, destaca-se a importância desse conhecimento anatómico para a actuação conservadora, visando o uso de métodos adequados e eficazes na avaliação, tratamento e reabilitação de pacientes com lesões no nervo isquiático, assim como para elaboração de acções preventivas.

As raízes ( $L 4$ - S3) que formam o nervo isquiático podem sofrer danos por factores variados: hérnia discal (compressão das raízes nervosas pelo disco intervertebral); espondilolistese (luxação dos corpos vertebrais que gera compressão das raízes nervosas); traumas mecânicos, ${ }^{22,23}$ inflamação, ${ }^{24}$ processo infeccioso ou tumoral, ${ }^{25,26}$ respostas químico-irritativas exacerbadas, ou a hipótese clínica mais rara, de compressão isquiática por varicosidades glúteas. ${ }^{27-29} \mathrm{O}$ conhecimento dos aspectos morfológicos e do desenvolvimento embrio-fetal do nervo isquiático são importantes para a avaliação subjectiva (anamnese) e cinéticofuncional (por provas de função específicas para avaliação do nervo isquiático ou diagnóstico diferencial da síndrome do piriforme).

P.S. Issack alerta-nos em 2009 para a crescente incidência $(30 \%)$ de lesões traumáticas do nervo isquiático, em relação com o aumento da prevalência moderna de fracturas acetabulares e de artroplastia da anca. ${ }^{23}$ Esse tipo de lesões pode resultar de lesão incisa do nervo, ou laceração por esquírolas de fractura óssea; de lesão por estiramento inerente às manobras ortopédicas da artroplastia coxo-femoral; ou por complicações pós-operatórias, como hematoma compressivo do trajecto do nervo.

Segundo Papadopoulos e Khan, ${ }^{30}$ a síndrome do piriforme foi primeiro descrita por Robinson em 1947, ${ }^{31} \mathrm{e}$ corresponde a compressão ou pinçamento do nervo isquiático por hipertrofia ou contractura do músculo piriforme, ou ainda por possíveis variações anatómicas do músculo. O quadro clínico caracteriza-se por alterações sensitivas, motoras e/ou tróficas da área de inervação do nervo isquiático. Segundo Ortiz Sanchez et al, ${ }^{32}$ essa síndrome pode constituir até $6,5 \%$ das causas de dor lombar, dor glútea ou dos restantes segmentos do membro inferior. Ainda segundo esses autores, a cirurgia deve reservar-se como último recurso para os casos em que falham todas as possíveis modalidades de tratamento conservador. Os conhecimentos prévios sobre a anatomia do nervo isquiático permitem elaborar planos de reabilitação específicos, adequados ao grau de compromisso e às necessidades funcionais de cada caso. Destaca-se a importância da relação entre o nervo isquiático e os músculos circundantes. Para além desta síndrome, Philipe Rigoard aponta ainda como causa não-discogénica de ciatalgia periférica, de natureza compressiva, a síndrome do músculo obturador interno, com afecção simultânea dos nervos isquiático e pudendo. ${ }^{33}$

\section{MATERIAL E MÉTODOS}

Material cadavérico humano: Todo o material aqui apresentado é resultado do trabalho de cuidadosa dissecção efectuada em cadáveres humanos legalmente doados e previamente embalsamados, tal como é prática corrente no laboratório de anatomia da NOVA Medical School, segundo a técnica apresentada por Goyri-O'Neill em 2013. ${ }^{14}$

Para o presente estudo foram utilizados dois cadáveres com a numeração 0042 e 0071 da listagem de doação cadavérica: Ambos do sexo feminino, o primeiro falecido com a idade de 89 anos e o segundo, de 66 anos.

Ambos foram embalsamados em 2016, um ano antes da dissecção para o presente estudo, e mantidos em câmaras de alta congelação $\left(-20\right.$ a $\left.-30^{\circ} \mathrm{C}\right)$. Por norma corrente do nosso departamento, ambos os cadáveres foram, previamente manipulados em diversos cursos de treino cirúrgico e laparoscópicos para fins de pós-graduação.

No cadáver 0071, detectou-se interessante variação anatómica, no decorrer das oito sessões de dissecção da disciplina de Anatomia Regional II. O segundo cadáver foi posteriormente utilizado como controlo, para dissecção comparativa, no estudo da variação anatómica bilateral do nervo isquiático.

\section{RESULTADOS}

Membro inferior esquerdo (variação anatómica): Em abril de 2017, no decurso das aulas regulares de dissecção cadavérica humana da NOVA Medical School, fomos 
interpelados por dois alunos que detectaram um caso de variação anatómica da origem, trajecto e divisão do nervo isquiático esquerdo de um cadáver do sexo feminino, com 66 anos de idade. No caso em análise, verificou-se, por dissecção e rebatimento dos músculos glúteo máximo e glúteo médio, a origem do tronco principal do nervo isquiático a um nível marcadamente mais baixo do que o frequente, sendo visíveis abaixo do músculo piriforme, quatro raízes de proveniência sagrada, envolvidas por baínha comum, e apenas convergentes a nível da tuberosidade isquiática, já cobertos pela musculatura glútea (Fig. 2A).

Após um curto trajecto de cerca de $8 \mathrm{~cm}$, o tronco principal do nervo emergia superficialmente, a nível da prega glútea, para logo se dividir, profundamente ao ponto de inserção do músculo bicípete femoral (Fig. 3A).

Membro inferior direito (variação anatómica): Em maio e junho de 2017, aproveitando o ensejo da visita de dois anatomistas da Universidade de Pernambuco, para treino de aperfeiçoamento técnico em dissecção cadavé- rica humana, constituiu-se grupo de trabalho para aprimorar a dissecção efectuada no membro inferior esquerdo, completando o trabalho com dissecção cuidadosa de todo o membro contralateral do mesmo cadáver. Completou-se esse estudo por dissecção pelas mesmas técnicas, num terceiro espécime de nervo isquiático, utilizado neste estudo como controlo comparativo.

No membro inferior direito desse mesmo cadáver feminino de 66 anos, observou-se origem e divisão do nervo isquiático, sobreponíveis ao anteriormente verificado no membro contra-lateral: divisão alta a nível do terço superior da coxa, após um curto trajecto, profundamente aos músculos glúteos. Nesse trajecto do nervo, observa-se, de modo distinto, no interior da baínha isquiática, separação das fibras constituintes do nervo, e a artéria isquiática. (Fig. $3 C)$

Membro inferior esquerdo (caso-controlo): Em junho de 2017, na sequência destas originais observações em ambos os membros do cadáver 0071, pareceu-nos

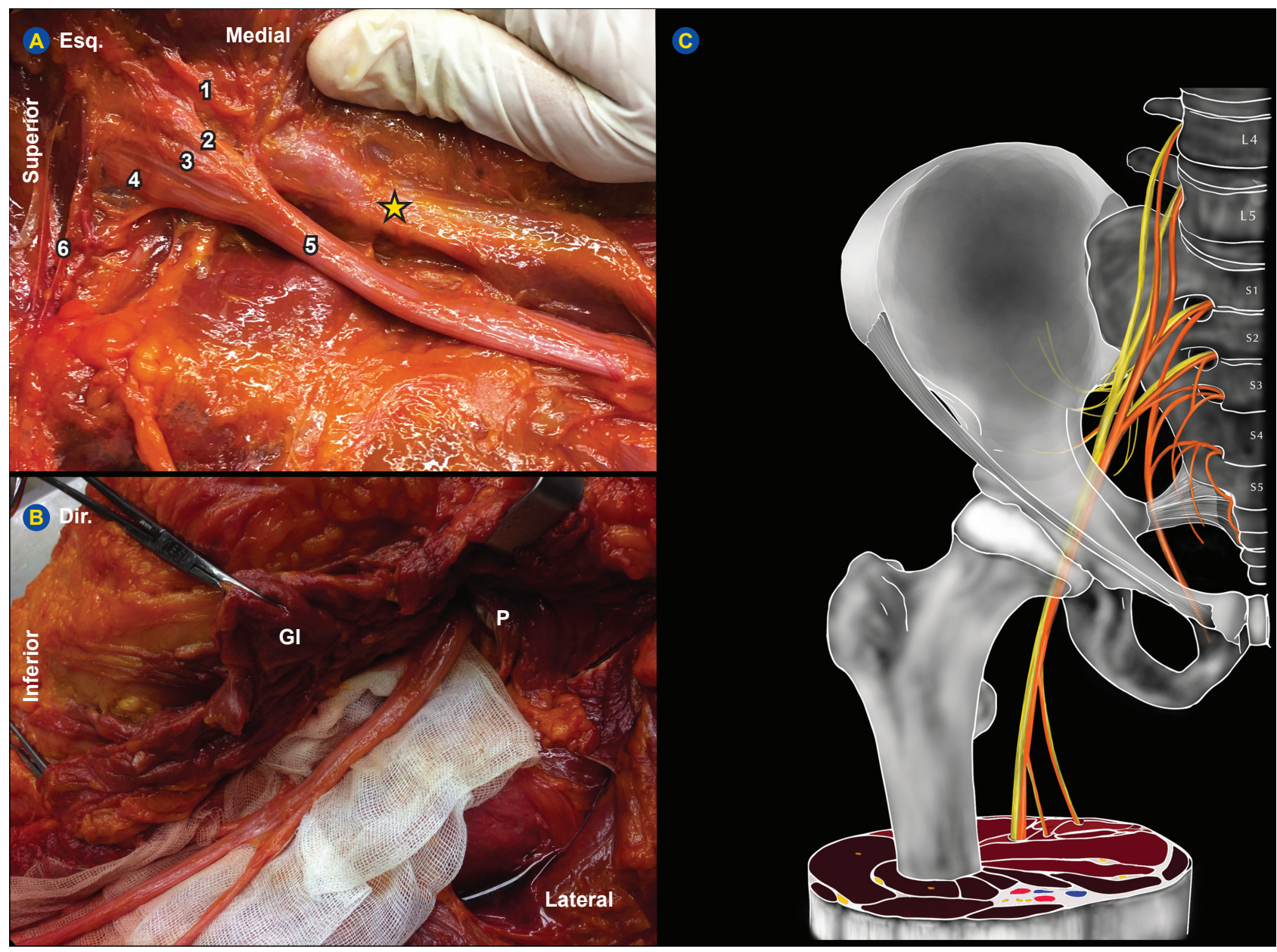

Figura 2 - Origem do nervo isquiático, dissecado bilateralmente, em cadáver do sexo feminino, de 66 anos: A: Membro inferior esquerdo - Na imagem A, verifica-se origem baixa do nervo (5), por união das raizes do plexo lombo-sagrado $(1 ; 2 ; 3 ; 4)$, após origem dos nervos glúteos superior e inferior (6), observada profundamente na região glútea, até ao nível da tuberosidade isquiática ( direito - A fotografia B documenta a dissecção efectuada no membro contralateral, em que se verifica origem do nervo abaixo do musculo Piriforme $(P)$ e curto trajecto de cerca de $10 \mathrm{~cm}$, até ao nível da tiberosidade isquiática, onde se observam caminhando lado a lado, as fibras dos nervos tibial e fibular comum, acompanhadas pela artéria isquiática, sendo o conjunto envolvido pela bainha isquiática. C: Versão artística da origem do nervo isquiático, por Daniel Casanova-Martinez (Chile), 2017. 


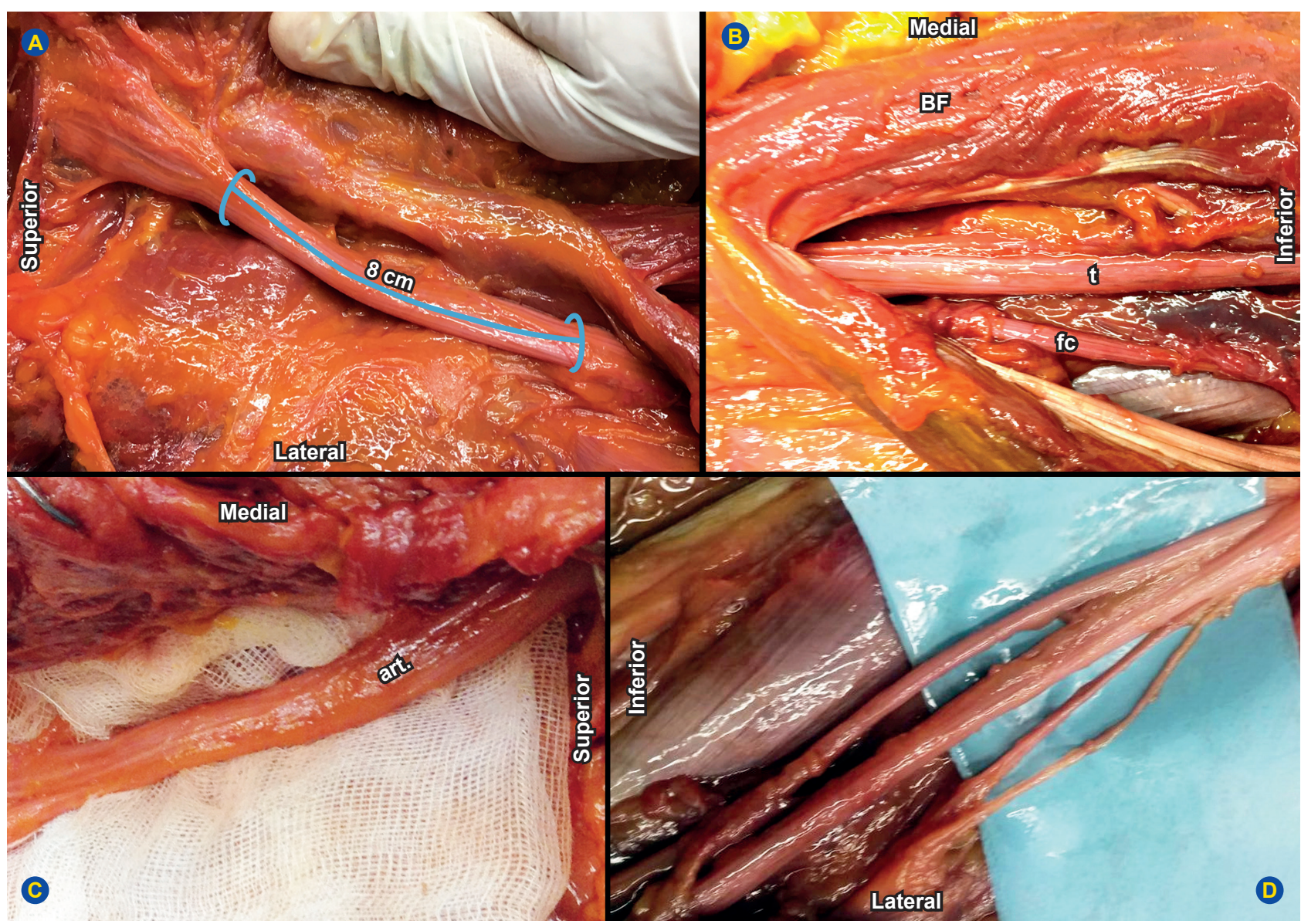

Figura 3 - Trajecto e divisão do nervo isquiático, dissecado bilateralmente, em cadáver do sexo feminino, de 66 anos. Do lado esquerdo, dissecou-se o tronco principal do nervo $(A)$, totalmente coberto pela musculatura glútea, num trajecto de cerca de $8 \mathrm{~cm}$, logo dividido a nível da tuberosidade isquiática, profundamente à inserção do músculo bicípete femoral (B "BF"), para fornecer os nervos Tibial (B "t") $e$ Fibular comum (B "fc"). Do lado direito, observou-se igualmente divisão alta do nervo, um pouco mais abaixo a nível do terço superior da face dorsal da coxa, profundamente aos musculos isquio-tibiais. Do mesmo modo que no membro esquerdo, observou-se trajecto das fibras nervosas, sempre divididas no interior da baínha isquiática, em conjunto com a artéria isquiática (C "art"). Após remoção cuidadosa da baínha envolvente, foi possível isolar o conjunto de ramos de divisão do nervo fibular comum, a nível poplíteo, bem visíveis na imagem D, rememorando, estranhamente, a proposta de Da Cortona de há 400 anos atrás (Fig. 1B).

pertinente reproduzir as mesmas técnicas de estudo num terceiro caso, utilizado como padrão. Procedeu-se a dissecção, pelo mesmo método, no cadáver 0042, igualmente do sexo feminino, embalsamado e conservado pela mesma técnica, um ano antes do estudo. Esse terceiro caso exibia nervo isquiático com origem, trajecto e relações, em tudo sobreponíveis à descrição mais corrente, e ao tipo classificativo mais frequente (85\%), de Beaton e Anson. ${ }^{21}$

\section{DISCUSSÃO}

O nervo isquiático é convencionalmente descrito

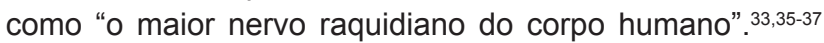
Interessou-nos portanto, de modo particular a análise do presente caso de variação anatómica bilateral que nos conduz a pensar que estamos em presença de um dos mais 'curtos' exemplares descritos na literatura médica, a par de um outro caso precedente, apresentado pelos mesmos co-autores, em 2013. ${ }^{1}$

K. Natsis et al (2014) demonstram, por dissecção de 147 casos, a importância cirúrgica do conhecimento das variações anatómicas do nervo isquiático, em relação com o músculo piriforme, enfatizando os casos infrequentes $(6,4 \%)$, em que apenas o nervo tibial nasce abaixo da margem inferior desse músculo. ${ }^{37}$

Ao aprofundar cuidadosamente a revisão bibliográfica, por consulta das mais antigas descrições anatómicas, encontramos os casos apresentados por Leonardo da Vinci, ${ }^{15}$ ou Pietro Da Cortona,${ }^{17}$ em que se verifica a detecção precoce, de modo 'visionário', de que o tronco do nervo isquiático seria apenas raramente 'indiviso'... No interior da baínha isquiática envolvente, caminhariam já divididas em dois ramos, as fibras dos nervos tibial e fibular comum, apenas unidos pela bainha conjuntiva que os envolve, em conjunto com o trajecto da artéria isquiática; Os dois conjuntos de fibras nervosas apenas se individualizam por separação, a altura variável entre a origem sagrada e o ápice da região poplítea, de acordo com a descrição dos anatomistas $\mathrm{Ph}$. Sappey ${ }^{19}$ ou J. Cruveilhier. ${ }^{20}$

No caso extremo de origem baixa e divisão alta do nervo isquiático, encontra-se em teoria, o caso de agenésia do 
nervo. Tal encontra-se descrito e devidamente comprovado por P. Coelho et al em 2013. ${ }^{38}$

O trabalho classificativo de Beaton e Anson ${ }^{21}$ tem sido adoptado pela grande maioria dos autores, como bem patente no extenso artigo de revisão assinado por $\mathrm{N}$. Apaydin em 2016, na Enciclopédia Bergman de Variações Anatómicas. ${ }^{34}$ Este refere, tal como já descrito por Sappey e Cruveilhier, que o ponto de divisão do nervo isquiático, para originar os dois ramos terminais (nervos tibial e fibular comum), é muito variável, podendo mesmo cada um destes ter origem separada como ramos directos do plexo sagrado. Refere ainda que os dois ramos terminais do nervo isquiático podem ser encontrados como dois nervos distintos ao longo de todo o trajecto do nervo isquiático, em cerca de $11 \%$ dos casos, envolvidos por uma bainha que, uma vez dissecada, permite seguir o trajecto de cada um dos nervos até aos respectivos ramos de origem, a nível sagrado. Por sua vez, ao analisar as causas de ciatalgia não-discogénicas, A. Mallikarjun e V. Sangeetha ${ }^{39}$ reportam por revisão da literatura médica entre 1997 e 2011, diversos casos apresentados de divisão alta de nervos isquiáticos, encontrando percentagens elevadas $(4,0 \%-20,9 \%)$ de divisão do nervo isquiático a nível pélvico, comparando a sua própria série de $6 \%$ de casos de divisão a nível pélvico.

A síndrome do piriforme, como causa do quadro clínico de ciatalgia, continua actualmente a suscitar estudos internacionais e tentativas de modernização da abordagem clínica. ${ }^{40,41,42}$

A hipótese de maior frequência de agenésia ou trajecto curto do tronco do nervo isquiático pode colocar importantes ilações clínicas e cirúrgicas, ${ }^{39}$ como no caso de bloqueios anestésicos, ${ }^{37,44}$ na terapêutica conservadora da ciatalgia, ${ }^{45}$ e também sobretudo nos procedimentos técnicos e seguimento clínico de casos de artroplastia da anca, ${ }^{36,39,43-45}$ cujo número crescente se deve ao envelhecimento gradual da população, para além do crescimento da cobertura populacional mundial em termos de cuidados de saúde.

A recente tendência para o sedentarismo da população, assumindo maior permanência preferencial em postura sentada, poderia por 'compressão' repetitiva das regiões posteriores da coxa e região glútea, ser co-responsável pelo recente aumento de prevalência de casos de divisão alta do nervo isquiático, tantas vezes assinalada na literatura médica mundial, em recentes anos (ainda no ano de 2017 detectámos mais três casos de divisão alta do isquiático, em oito cadáveres dissecados).

Relativamente às técnicas e materiais utilizados para o estudo, será importante enfatizar o reconhecimento internacional da originalidade e modernização factual das técnicas de conservação cadavérica do Departamento de Anatomia da NOVA Medical School.

A técnica de perfusão intermitente de dietilenoglicol permite manutenção da aparência de frescura para dissecção, mesmo vários meses após embalsamamento, incluindo casos em que o mesmo cadáver é utilizado para diversos cursos pós-graduados ou de treino cirúrgico laparoscópico, previamente à sua utilização para dissecção com âmbito de investigação ou pesquisa anatómica. $O$ facto de não ser acrescentado formaldeído ao líquido de embalsamamento permite várias horas de dissecção sem o transtorno de odores nem preocupações pela saúde dos manipuladores. O autor Balta, ${ }^{46}$ estabelecendo em 2015 um estudo comparativo das diversas técnicas de embalsamamento e conservação cadavérica ao longo dos séculos, coloca a nossa técnica no topo da listagem, como mais inovadora e aperfeiçoada. Em 2017, Nazreen Shariff e Alia Amin ${ }^{47}$ retomam o assunto, reportando que as nossas técnicas permitem a conservação cadavérica por mais de um ano. Verificamos, porém na prática, boa conservação por mais de cinco anos. Na sequência da introdução, por Esperança Pina, da prática de perfusão arterial de dietilenoglicol, em substituição do formaldeído, Goyri-O'Neill tem vindo a desenvolver e aperfeiçoar a qualidade de impregnação dos tecidos por introdução de um mecanismo de perfusão arterial intermitente, por bomba perfusora integrada em sistema informático patenteado que permite regular fluxos e pressões de acordo com o índice de massa corporal (IMC) individual.

\section{CONCLUSÃO}

Diversas ilações são possíveis, por revisão do presente caso de variação anatómica bilateral infrequente do nervo isquiático, para além da indiscutível importância clínica e cirúrgica.

Por mais meticulosos que sejam os nossos estudos anatómicos, jamais poderemos considerar terminada ou definitiva, a investigação em anatomia humana. Novos campos de conhecimento, e novas oportunidades de pesquisa, vão-se oferecendo a cada vez que aprofundamos o conhecimento anatómico.

Não deverão ser negligenciados os esforços dos que ensinam e investigam a matéria anatómica e a arte da dissecção. O valor inestimável do estudo anatómico fundamenta plenamente a investigação e desenvolvimento de uma melhor prática clínica.

Nesse sentido, e com base nas ilustrações do presente caso anatomo-clínico, afigura-se-nos de primordial importância a manutenção dos esforços de melhor desenvolvimento da técnica e da prática da dissecção cadavérica humana, tanto para melhor qualidade dos estudos médicos pré-graduados, como ainda para aperfeiçoamento da qualidade técnica dos estudos pós-graduados e de treino cirúrgico, contribuindo para a melhor fundamentação do raciocínio em investigação clínica e cirúrgica.

\section{AGRADECIMENTOS}

Formulamos especial agradecimento aos docentes Ana Filipa Palma dos Reis Pereira e João Mendes, e aos discentes Ana Sofia Oliveira e Ruben Rodrigues, pela detecção e documentação fotográfica do primeiro caso de variação anatómica, no decorrer das aulas de dissecção da disciplina de Anatomia Regional II, na NOVA Medical School, Lisboa.

A Vyacheslav Sushchyk (Slava) e José Carreira, pelo imprescindivel apoio técnico ao embalsamamento e 
dissecção.

E a Teresa Rodrigues de Sousa, pelo seu essencial empenho na manutenção do sistema de listagem e doação cadavérica, proporcionando a extensa listagem de perto de 3000 doações legalizadas.

Muitos dos dadores anónimos da nossa listagem encontram-se ainda vivos, mantendo estreita relação com Teresa Rodrigues de Sousa. A todos eles, formulamos especial nota de apreço e gratidão pela generosa dádiva.

\section{PROTECÇÃO DE PESSOAS E ANIMAIS}

Os autores declaram que os procedimentos seguidos estavam de acordo com os regulamentos estabelecidos pelos responsáveis da Comissão de Investigação Clínica e Ética e de acordo com a Declaração de Helsínquia da

\section{REFERÊNCIAS}

1. Pais D, Casal D, Bettencourt Pires MA, Furtado A, Bilhim T, Almeida MA et al. Sciatic nerve high division: two different anatomical variants with important clinical implications. Acta Med Port. 2013;26:208-11.

2. Bettencourt Pires MA, Esperança Pina JA. Os esfolados. Anatomia, dissecção e modelos anatómicos. [consultado 2018 mai 19]. Disponível em http://www.sociedadeanatomica.pt/site/upload/files/ fich_99218405585_foma-editada--archives-of-anatomy.pdf.

3. Bettencourt Pires MA. Interdisciplinarity, multiculturalism. anatomy and art. UPE, Brasil. Agosto 2017. [consultado 2018 mai 19]. Disponível em: https://www.researchgate.net/publication/319332280_ Interdisciplinarity_Multiculturalism_Anatomy_and_Art.

4. Prakash, Prabhu LV, Rai R, D'Costa S, Jiji PJ, Singh G. Cadavers as teachers in medical education: knowledge is the ultimate gift of body donors. Singapore Med J. 2007;48:186-9.

5. Bergman EM, Prince KJ, van der Vieuten CP, Scherpbier AJ. How much anatomy is enough? Anat Sci Ed. 2008;1:184-8.

6. Moxham BJ, Shaw H, Crowson R, Plaisant O. The future of clinical anatomy. Eur J Anat. 2011;15:29-46.

7. Brenner E, Pais D. Philosophy and ethics of anatomy teaching. Eur $\mathrm{J}$ Anat. 2014;18:353-60.

8. Cunha Júnior, IF, Cunha de Sousa Filho G, Bezerra Cavalcante A, Pacífico FA. É possível utilizar o Código Civil para regular a doação cadavérica post mortem? Rev Acad Fac Direito Recife. 2015;87:163-78.

9. Moraes GN, Falcão JG, Sandes AA, Nascimento IY, Scwingel IP, Silva Júnior EX. Cadaveric dissection by students monitors in human anatomy discipline: experience report. J Morphol Sci. 2016;33:68-72.

10. Tubbs S. Why anatomy is neglected or mistaught. Clin Anat. 2017;30:1001.

11. Rocha AO, Farina Júnior MA, Girotto MC, Moraes MP, Thomaz GD, Campos D, et al. The Brazilian Ceremony in Honor of Body Donors: an opportunity to express gratitude and reflect on medical education. Int J Innov Educ Res. 2018;6:264-73.

12. Goyri-O'Neill JE, Águas A, Madeira D, Gonçalves-Ferreira A, Carvalho Sousa N, Miguéis A.C, et al. Coordination and training across Portugal is enhancing the quality of undergraduate and postgraduate medical courses. Clin Anat. 2014;27:940.

13. Pais D, Casal D, Lemos LM, Barata P, Moxham BJ, Goyri-O'Neill J. Outcomes and satisfaction of two optional cadaveric dissection courses: a 3-year prospective study: Optional Cadaveric Dissection Courses. Anat Sci Educ. 2017;10:127-36.

14. Goyri-O'Neill J, Pais D, Freire de Andrade F, Ribeiro P, Belo A, O'Neill $M A$, et al. Improvement of the embalming perfusion method: the innovation and the results by light and scanning electron microscopy. Acta Med Port. 2013;26:188-94.

15. da Vinci L. Esboços do nervo isquiático. Windsor Collection-Na.B.f.18/ Clark1903 5 r/c. 1490-1492 e Windsor Collection - Q IV f. 9 r/Clark 19114 r/c.1495-1499. In: Huard P. (1968) Léonard de Vinci. Dessins Anatomiques. Paris: Dacosta; 1968.

16. Paré A. Le sixième livre d'anatomie. Buon G, Éditeur. 1585. In: Oeuvres complètes d'Ambroise Paré de la Val du Maine. $4^{\text {ème }}$ éd. Paris: Éditions Louis Pariente; 1969. p. 94 e 222.

17. Da Cortona P. Tabulae anatomicae, Tab.XIX, 1618. In: Dover Pictorial Archive Series. Classical Anatomical Illustrations. New York: Dover;
Associação Médica Mundial.

\section{CONFIDENCIALIDADE DOS DADOS}

Os autores declaram ter seguido os protocolos do seu centro de trabalho acerca da publicação de dados. Consentimento do doente obtido.

\section{CONFLITOS DE INTERESSE}

Todos os autores declaram a ausência de conflitos de interesse.

\section{FONTES DE FINANCIAMENTO}

Os autores declaram não ter recebido subsídios ou bolsas para a elaboração do artigo.

2008.

18. Bartolommeo Eustachius, Opuscula Anatomica, Tab.XX, 1564, In: Dover Pictorial Archive Series. Classical Anatomical Illustrations. New York: Dover; 2008.

19. Sappey Ph. C. Névrologie. In: Traité d'anatomie descriptive, T.III, Fig.557. Paris: Delahaye; 1872. p. 455-6.

20. Cruveilhier J. Angéologie et névrologie. In: Traité d'anatomie descriptive. $4^{\text {ème }}$ éd. Asselin et Labé: Paris ; 1871. p. 652.

21. Beaton $L, A$ nson $B$. The relation of the sciatic nerve and of its subdivisions to the pryriformis muscle. Anat Rec. 1937;70:1-5.

22. Maripuu A, Björkman A, Björkman-Butscher IM, Mannfold P, Andersson $G$, Dahlin LB. Reconstruction of sciatic nerve after traumatic injury in humans - factors influencing outcome as related to neurobiological knowledge from animal research. J Brachial Plex Peripher Nerve Inj. 2012;7:2-13.

23. Issack PS, Helfet DL. Sciatic nerve injury associated with acetabular fractures. HSSJ. 2009;5:12-8.

24. Kouzaki K, Nakazato K, Mitsuno M, Yonechi T, Higo Y, Kubo Y, et al. Sciatic nerve conductivity is impaired by hamstring strain injuries. Int $\mathrm{J}$ Spots Med. 2017. Sept.11. [consultado 2018 mai 19]. Disponível em: [https://www.thieme-connect.com/DOI/DOI?10.1055/s-0043-115735].

25. Woo PY, Ho JM, Mak CH, Wong AK, Wong HT, Chan KY. A rare cause of sciatica discovered during digital rectal examination: case report of an intrapelvic sciatic notch schwannoma. Br J Neurosurg. 2017;14:1-4.

26. Nwankwo BO, Henshaw RM, Kumar D. Glomus tumor of the sciatic nerve: an extraspinal cause of sciatica. Orthopedics. 2017:1-3.

27. Bendszus M, Rieckmann P, Perez J, Koltzenburg M, Reiners K, Solymosi L. Painful vascular compression syndrome of the sciatic nerve caused by gluteal varicosities. Neurology. 2003;61:985-7.

28. $\mathrm{Hu} \mathrm{MH}$, Wu KW, Jian YM, Wang CT, Wu IH, Yang $\mathrm{SH}$. Vascular compression syndrome of sciatic nerve caused by gluteal varicosities. Ann Vasc Surg. 2010;24:1134.

29. Zhang Z, Zhang X, Yang C, Wen X. Refractory sciatica caused by gluteal varicosities. Orthopade. 2017;46:781-4.

30. Papadopoulos EC, Khan SN. Piriformis syndrome and low back pain: a new classification and review of the literature. Orthop Clin North Am. 2004;35:65-71.

31. Robinson DR. Pyriformis syndrome in relation to sciatic pain. Am J Surg. 1947;73:335-58.

32. Ortiz Sánchez VE, Charco Roca LM, Soria Quiles A, Zafrilla Disla E, Hernandez Mira F. Síndrome piramidal y variaciones anatómicas como causa de dolor ciático insidioso. Rev Esp Anestesiol Reanim. 2014;61:521-4.

33. Rigoard P. The sciatic nerve. In: Rigoard's Atlas of the Peripheral Nerves. 2017. p. 224-43.

34. Apaydin N. Lumbosacral plexus. In: Bergmann's Comprehensive Encyclopedia of Human Anatomic Variation. Chap. 92. Tubbs RS, Shoja MM, Loukas M, editors. Berlin: Wiley Sons; 2016. p. 1121-2.

35. Prakash, Bhardwaj AK, Devi MN, Sridevi NS, Rao PK, Singh G. Sciatic nerve division: a cadaver study in the Indian population and review of the literature. Singapore Med J. 2010;51:721-3.

36. Banon S, Itoo MS, et al. Higher division of sciatic nerve and its clinical importance. Int J Basic Appl Sci. 2014;3:23-5. 
37. Natsis K, Totlis T, Konstantinidis GA, Paraskevas G, Piagkou M, Koebke $\mathrm{J}$. Anatomical variations between the sciatic nerve and the piriformis muscle: a contribution to surgical anatomy in piriformis syndrome. Surg Radiol Anat. 2014;36:273-80.

38. Coelho P, Melo C, Bernardes A. Rare anatomical variation of absence of the sciatic nerve: completely substituted by the tibial and common fibular nerve. Acta Med Port. 2013;26:283-6.

39. Mallikarjun A, Sangeetha V. Study on variant anatomy of sciatic nerve. J Clin Diagn Res. 2014;8:AC07-9.

40. Olsen W, Elias M. A rare cause of piriformis muscle syndrome. Pain Clin. 2000;12:117-9.

41. Dufour X, Evelinger S, Cerioli A. Point d'anatomie. Focus sur le syndrome du piriforme: étiologie, test et niveaux de preuve. L'anatomie au service de la clinique. Kinesither Rev. 2017; 17: 188-9.

42. Hopayan K, Danielyan A. Four symptoms define the piriformis syndrome: an updated systematic review of its clinical features. Eur J Orthop Surg Traumatol. 2018;28:155-64.

43. Haladaj R, Pingot M, Polguj M, Wysiadecki G, Topol M. Anthropometric study of the piriformis muscle and sciatic nerve: a morphological analysis in a Polish population. Med Sci Monit. 2015:21:3760-68.

44. Kanawati AJ. Variations of the sciatic nerve anatomy and blood supply in the gluteal region: a review of the literature. ANZ J Surg. 2014;84:816-9.

45. Willis-Owen CA, Nishiwaki T, Spriggins AJ. Sciatic palsy after total hip arthroplasty associated with vascular graft occlusion. Hip Int. 2011;21:118-21.

46. Balta JY, Cronin M, O'Maqhony SM. Human preservation techniques in anatomy: a $21^{\text {st }}$ century medical education perspective. Clin Anat. 2015;28:725-34

47. Shariff N, Amin A. Comparative study of long term preservation of human cadaveric tissue. World J Pharm Res. 2017;6:172-84. 